\title{
O USO DE POLÍMEROS NO TRATAMENTO E RECUPERAÇÃO DE FLUIDOS DE PERFURAÇÃO CONTAMINADOS
}

\author{
USE OF POLYMERS FOR TREATING AND RECOVERING \\ OF CONTAMINATED DRILLING FLUIDS
}

\author{
L.V. Amorim; M.I.R. Barbosa; J.D. Viana; K.V. Farias; E. Pereira; H.C. Ferreira
}

\begin{abstract}
Recebido em:17/12/2004; aceito em: 16/09/2005
RESUMO O objetivo deste trabalho é avaliar o efeito de aditivos poliméricos celulósicos e não-celulósicos visando a proteção e reabilitação de fluidos hidroargilosos com diferentes graus de contaminação. Foram estudadas duas amostras de argilas bentoníticas sódicas e três aditivos poliméricos, sendo uma amostra de poliacrilamida (PHP), uma amostra de carboximetilcelulose de baixa viscosidade (CMC BV) e uma amostra composta pela mistura destes polímeros (MIX). Os fluidos foram preparados com concentração de 4,86\% em massa de argila. Para o processo de proteção, os fluidos foram tratados com os aditivos poliméricos e após $24 \mathrm{~h}$, contaminados com $\mathrm{CaCl}_{2}+\mathrm{MgCl}_{2}$ em diferentes teores. $\mathrm{O}$ processo de reabilitação foi realizado com a introdução dos aditivos em ordem inversa. A avaliação dos processos foi realizada por meio da determinação das viscosidades aparente e plástica, em viscosímetro Fann 35A, e do volume de filtrado em filtro prensa Fann. Os resultados mostraram a eficiência do CMC BV e do MIX na proteção e reabilitação dos fluidos contaminados com diferentes concentrações de $\mathrm{CaCl}_{2}+\mathrm{MgCl}_{2}$.
\end{abstract}

Palavras chave: Fluidos hidroargilosos, contaminação, aditivos poliméricos.

\begin{abstract}
The aim of this work is to evaluate the effect of cellulose and non-cellulose polymeric additives for protection and recovery of clay and water based drilling fluids with different contamination degrees. Two samples of sodium bentonite clay and three polymeric additives - PHP, polyacrilamide, CMC BV, carboximethylcellulose of low viscosity and MIX, mixture of PHP and CMC BV - were studied. For the protection process, the drilling fluids prepared with $4.86 \% \mathrm{w} / \mathrm{w}$, were treated with polymeric additives and after 24 hours, contaminated with $\mathrm{CaCl}_{2}+\mathrm{MgCl}_{2}$ (degradation additive). The recovery process was made by addition polymeric additives in inverse order. The apparent and plastic viscosities and water loss were measured by using viscosimeter Fann 35A and filter press Fann, respectively. The results show the efficiency of CMC BV and MIX in the protection and recovery of drilling fluids contaminated with $\mathrm{CaCl}_{2}+\mathrm{MgCl}_{2}$ in different concentrations.
\end{abstract}

Keywords: contamination, drilling fluids, polymeric additives

\section{INTRODUÇÃO}

Estudos envolvendo o efeito de aditivos poliméricos em fluidos de perfuração hidroargilosos vêm sendo realizados na Universidade Federal de Campina Grande (UFCG), antigo Campus II da Universidade Federal da Paraíba, desde 1991. Os primeiros estudos avaliaram o efeito da adição de lignosulfonatos, lignitos e polímeros à base de celulose, comercialmente conhecidos por Spersene, XP-20 e CMC, respectivamente, sob a reologia de dispersões de argilas bentoníticas sódicas (PEDROSO, 1991; ACCIOLY, 1994).

Em 2002, foram iniciados estudos com o objetivo de avaliar a ação protetora e de recuperação de aditivos poliméricos em fluidos hidroargilosos contaminados com cloretos de cálcio e magnésio.
Esta linha de pesquisa foi motivada pelos problemas de contaminação de fluidos frequientemente enfrentados durante as operações de perfuração de poços de petróleo, poços tubulares e de sondagem. Os primeiros experimentos foram realizados com fluidos preparados com argilas bentoníticas sódicas industrializadas na Paraíba e tratados com uma amostra de carboximetilcelulose (CMC) de baixa viscosidade. Os resultados indicaram a viabilidade do uso do CMC como agente de proteção e recuperação de fluidos hidroargilosos (AMORIM et al., 2002). A partir daí, diversos trabalhos foram desenvolvidos utilizando diferentes variedades de argilas bentoníticas provenientes dos depósitos localizados no Município de Boa Vista, (PB), e CMCs com diferentes graus de viscosidade (AMORIM et al., 2003; AMORIM et al., 2004; FARIAS, 2003).

${ }^{1}$ DEMa/CCT/Universidade Federal de Campina Grande (luciana@labdes.ufcg.edu.br); (heber@dema.ufcg.edu.br); (ingrid@labdes.ufcg.edu.br); (josiane@labdes.ufcg.edu.br); (kassievieira@bol.com.br)

${ }^{2}$ System Mud Indústria e Comércio Ltda (eugenio@systemmud.com.br) 
Também como parte desta linha de pesquisa, foram estudadas amostras de CMC e PAC (celulose polianiônica) em fluidos à base de água e argilas bentoníticas com diferentes graus de contaminação. Os resultados alcançados com este estudo mostraram a eficiência destes polímeros como colóide protetor também para fluidos contaminados com elevadas concentrações de cloretos de cálcio e magnésio (BARBOSA et al., 2004a; BARBOSA et al., 2004b).

Como continuidade, objetiva-se com este trabalho avaliar o efeito de polímeros celulósicos e não-celulósicos visando a proteção e reabilitação de fluidos de perfuração hidroargilosos contaminados com diferentes teores dos cloretos de cálcio e magnésio.

\section{MATERIAIS}

\section{Argilas Bentoníticas}

Foram estudadas duas amostras de argilas bentoníticas sódicas, uma obtida em laboratório, denominada argila $\mathrm{A}$, e uma industrializada em Campina Grande (PB), denominada argila B, composta por uma mistura de argilas bentoníticas. As argilas estudadas, tanto a obtida em laboratório quanto as que compõem a argila industrializada, são provenientes de Boa Vista (PB).

\section{Aditivos Poliméricos}

Foram estudados três aditivos poliméricos: poliacrilamida, denominado de PHP, carboximetilcelulose de baixa viscosidade, denominado de CMC BV, e uma amostra composta pela mistura destes dois polímeros, denominada MIX. Os aditivos foram estudados nas concentrações de 0,3, 0,4 e 0,5 g / 24,3 g de argila seca. No texto, essas concentrações serão indicadas como: $0,3 \mathrm{~g}, 0,4 \mathrm{~g}$ e $0,5 \mathrm{~g}$. Na Tabela 1 , estão apresentados os dados técnicos dos aditivos.

Os aditivos foram fornecidos pela Empresa System Mud Indústria e Comércio Ltda..

\section{Aditivo de Contaminação}

Foram utilizados como agente de contaminação o cloreto de cálcio para análise (P.A.) $\left(\mathrm{CaCl}_{2}\right)$ e cloreto de magnésio P.A. $\left(\mathrm{MgCl}_{2}\right)$ da marca VETEC. Esses aditivos foram adicionados simultaneamente nas concentrações de 10 meq de $\mathrm{CaCl}_{2} / 100 \mathrm{~g}$ de argila seca +10 meq de $\mathrm{MgCl}_{2} / 100 \mathrm{~g}$ de argila seca, 20 meq de $\mathrm{CaCl}_{2} / 100 \mathrm{~g}$ de argila seca +20 meq de $\mathrm{MgCl}_{2}$ $/ 100 \mathrm{~g}$ de argila seca e 30 meq de $\mathrm{CaCl}_{2} / 100 \mathrm{~g}$ de argila seca +30 meq de $\mathrm{MgCl}_{2} / 100 \mathrm{~g}$ de argila seca. No texto, essas concentrações serão indicadas como: 10 meq de $\mathrm{CaCl}_{2}+10$ meq de $\mathrm{MgCl}_{2}, 20$ meq de $\mathrm{CaCl}_{2}+20$ meq de $\mathrm{MgCl}_{2}$ e 30 meq de $\mathrm{CaCl}_{2}+30$ meq de $\mathrm{MgCl}_{2}$.

\section{METODOLOGIA}

\section{Preparação dos Fluidos de Perfuração}

Os fluidos de perfuração foram preparados com concentração de 4,86 \% em massa de argila (24,3 g de argila em $500 \mathrm{~mL}$ de água deionizada), de acordo com a norma N-2605 (PETROBRAS, 1998a).

\section{Tratamento de Proteção}

Após preparação, os fluidos foram tratados com os aditivos poliméricos e agitados durante 5 min. Após $24 \mathrm{~h}$, foram contaminados com os diferentes teores de $\mathrm{CaCl}_{2}+\mathrm{MgCl}_{2}$ e novamente agitados durante $5 \mathrm{~min}$, permanecendo em repouso por mais $24 \mathrm{~h}$. Em seguida, foram medidas as viscosidades aparente (VA) e plástica (VP), em viscosímetro Fann 35A e o volume de filtrado (VF), em filtro-prensa Fann, segundo a norma N-2605 (PETROBRAS, 1998a).

Tabela 1. Dados técnicos dos aditivos poliméricos

Table 1. Technical data of the polymeric additives

\begin{tabular}{|c|c|c|c|}
\hline Dados técnicos & CMC BV & PHP & MIX \\
\hline Função & $\begin{array}{l}\text { Controle de reboco e } \\
\text { filtrado }\end{array}$ & Viscosificante & $\begin{array}{l}\text { Viscosificante / controle de } \\
\text { reboco e filtrado }\end{array}$ \\
\hline Meio de aplicação & Água doce e salgada & Água doce e salgada & Água doce e salgada \\
\hline Composição & Carboximetilcelulose & Poliacrilamida & $\begin{array}{l}\text { Carboximetilcelulose e } \\
\text { poliacrilamida }\end{array}$ \\
\hline Tamanho de cadeia & Curta & Longa & Curta e longa \\
\hline $\begin{array}{l}\text { Grau de substituição } \\
\text { (DS) }\end{array}$ & 0,85 & $?$ & 0,85 \\
\hline Pureza $(\%)$ & $>97,50$ & $>98,00$ & $>97,50$ \\
\hline
\end{tabular}




\section{RESULTADOS E DISCUSSÃO}

Os resultados mostraram que a aditivação polimérica corrige o comportamento reológico dos fluidos preparados com a argila $\mathrm{A}$, sendo obtidos valores de VA, VP e VF de acordo com os limites especificados para uso na perfuração de poços de petróleo (PETROBRAS, 1998b). Os melhores resultados foram obtidos com a adição de $0,4 \mathrm{e}$ $0,5 \mathrm{~g}$ de CMC BV (Tabela 2) e 0,3 e $0,5 \mathrm{~g}$ de MIX (Tabela 6).

Tabela 2. Propriedades dos fluidos preparados com a argila A, aditivados com CMC BV, degradados e após os tratamentos de proteção e recuperação.

Table 2. Properties of drillings fluids prepared with clay called A, treated with CMC BV, and submitted to degrading additives and after treating of protection and recovery

\begin{tabular}{|c|c|c|c|c|}
\hline $\begin{array}{c}\text { CMC BV } \\
\text { (g/24,3g de argila) }\end{array}$ & $\begin{array}{c}\mathrm{CaCl}_{2}+\mathrm{MgCl}_{2} \\
\text { (meq/100g de argila) }\end{array}$ & (cP) & (cP) & $(\mathbf{m L})$ \\
\hline- & - & 12,5 & 4,8 & 17,7 \\
\hline 0,3 & - & 13,3 & 7,0 & 17,3 \\
\hline 0,4 & - & 15,5 & 8,0 & 17,2 \\
\hline 0,5 & - & 18,5 & 7,0 & 16,3 \\
\hline- & $10+10$ & 10,8 & 4,5 & 17,8 \\
\hline- & $20+20$ & 8,5 & 4,0 & 21,0 \\
\hline- & $30+30$ & 9,7 & 3,8 & 20,5 \\
\hline \multicolumn{5}{|c|}{ Tratamento de Proteção } \\
\hline \multirow{3}{*}{0,3} & $10+10$ & 15,3 & 7,0 & 17,5 \\
\hline & $20+20$ & 25,3 & 8,0 & 18,0 \\
\hline & $30+30$ & 31,5 & 4,0 & 17,4 \\
\hline \multirow{3}{*}{0,4} & $10+10$ & 17,3 & 7,0 & 16,0 \\
\hline & $20+20$ & 19,0 & 7,0 & 17,0 \\
\hline & $30+30$ & 20,5 & 7,0 & 18,0 \\
\hline \multirow{3}{*}{0,5} & $10+10$ & 20,5 & 7,0 & 15,3 \\
\hline & $20+20$ & 20,8 & 7,5 & 17,8 \\
\hline & $30+30$ & 21,3 & 7,0 & 17,5 \\
\hline \multicolumn{5}{|c|}{ Tratamento de Recuperação } \\
\hline $\begin{array}{c}\mathrm{CaCl}_{2}+\mathrm{MgCl}_{2} \\
\text { (meq/100g de argila) }\end{array}$ & $\begin{array}{c}\text { CMC BV } \\
\text { (g/24,3g de argila) }\end{array}$ & VA $(\mathbf{c P})$ & $\mathbf{V P}(\mathbf{c P})$ & VF (mL) \\
\hline $10+10$ & & 17,5 & 6,5 & 18,0 \\
\hline $20+20$ & 0,3 & 17,3 & 7,0 & 19,2 \\
\hline $30+30$ & & 14,5 & 6,0 & 21,3 \\
\hline $10+10$ & & 15,5 & 7,5 & 17,3 \\
\hline $20+20$ & 0,4 & 16,5 & 8,0 & 16,5 \\
\hline $30+30$ & & 16,5 & 7,0 & 17,5 \\
\hline $10+10$ & & 19,0 & 8,0 & 16,3 \\
\hline $20+20$ & 0,5 & 19,0 & 6,5 & 18,4 \\
\hline $30+30$ & & 19,3 & 8,0 & 17,5 \\
\hline \multicolumn{2}{|c|}{ Especificações (PETROBRAS, 1998b) } & $\geq 15,0$ & $\geq 4,0$ & $\leq 18,0$ \\
\hline
\end{tabular}

A aditivação polimérica promoveu aumento nas viscosidades e redução no volume de filtrado dos fluidos preparados com a argila A. Quando os aditivos poliméricos são adicionados aos fluidos hidroargilosos, estes são dispersos na fase líquida (água) fazendo com que a cadeia polimérica seja hidratada e assuma uma configuração alongada. Este alongamento da cadeia polimérica é responsável pelo aumento da viscosidade do sistema e redução do $\mathrm{VF}$, promovendo uma adsorção entre as cargas negativas do polímero e as cargas positivas presentes nas arestas das partículas de argila, neutralizando-as e fazendo com que ocorra uma repulsão mútua entre elas (AMORIM, 2003).

Segundo Oliveira et al. (2000), o comportamento apresentado pelos fluidos preparados com a argila A pode ser explicado através dos mecanismos de estabilização eletrostática e eletroestérica. A estabilização eletrostática ocorre como conseqüência da repulsão entre as cadeias do polímero adsorvidas às partículas de argila, enquanto que, a estabilização eletroestérica ocorre como 
consequiência do aumento da distância mínima de separação entre as partículas de argila que estão envolvidas pelas cadeias poliméricas e pelas interações de caráter elétrico repulsivo entre as cadeias do polímero, evitando a floculação do sistema.

Para os fluidos preparados com a argila B, observou-se que a aditivação polimérica elevou e muito os valores de VA, conduzindo o sistema ao estado floculado (Tabelas 3, 5 e 7). Os fluidos aditivados com PHP (Tabela 5) desenvolveram sistemas classificados como floculados-gel (estado no qual as partículas de argila estão arranjadas numa estrutura denominada de castelo-de-cartas) com valores de VA superiores a $100,0 \mathrm{cP}$. Este comportamento contradiz a discussão apresentada acima, porém, polímeros de cadeia longa, como a poliacrilamida (PHP), podem causar o fenômeno de floculação através dos efeitos de encapsulamento (PEREIRA, 2001), formação de pontes (SOMASUNDARAN et. al, 1996) e quando segmentos de uma mesma cadeia polimérica são adsorvidos às superfícies de diferentes partículas de argila (LUCKHAM; ROSSI, 1999).

Tabela 3. Propriedades dos fluidos preparados com a argila B, aditivados com CMC BV, degradados e após os tratamentos de proteção e recuperação

Table 3. Properties of drillings fluids prepared with clay called B, treated with $C M C B V$, and submitted to degrading additives and after treating of protection and recovery

\begin{tabular}{|c|c|c|c|c|}
\hline $\begin{array}{c}\text { CMC BV } \\
\text { (g/24,3g de argila) }\end{array}$ & $\begin{array}{c}\mathrm{CaCl}_{2}+\mathrm{MgCl}_{2} \\
\text { (meq/100g de argila) }\end{array}$ & VA (cP) & $\mathbf{V P}(\mathbf{c P})$ & VF (mL) \\
\hline- & - & 16,1 & 4,0 & 16,3 \\
\hline 0,3 & - & 20,8 & 7,0 & 14,8 \\
\hline 0,4 & - & 25,5 & 6,5 & 15,0 \\
\hline 0,5 & - & 27,8 & 6,5 & 15,0 \\
\hline- & $10+10$ & 16,8 & 3,5 & 19,8 \\
\hline- & $20+20$ & 11,8 & 3,0 & 27,5 \\
\hline- & $30+30$ & 5,0 & 2,5 & 39,0 \\
\hline \multicolumn{5}{|c|}{ Tratamento de Proteção } \\
\hline \multirow{3}{*}{0,3} & $10+10$ & 36,3 & 3,5 & 17,5 \\
\hline & $20+20$ & 38,8 & 2,5 & 19,7 \\
\hline & $30+30$ & 31,5 & 5,0 & 23,3 \\
\hline \multirow{3}{*}{0,4} & $10+10$ & 43,5 & 4,5 & 17,0 \\
\hline & $20+20$ & 46,8 & 4,0 & 20,0 \\
\hline & $30+30$ & 34,3 & 6,0 & 22,7 \\
\hline \multirow{3}{*}{0,5} & $10+10$ & 46,8 & 3,0 & 15,5 \\
\hline & $20+20$ & 50,5 & 5,5 & 18,5 \\
\hline & $30+30$ & 32,8 & 5,0 & 21,5 \\
\hline \multicolumn{5}{|c|}{ Tratamento de Recuperação } \\
\hline $\begin{array}{c}\mathrm{CaCl}_{2}+\mathrm{MgCl}_{2} \\
\text { (meq/100g de argila) }\end{array}$ & $\begin{array}{c}\text { CMC B V } \\
\text { (g/24,3g de argila) }\end{array}$ & VA (cP) & $\mathbf{V P}(\mathbf{c P})$ & VF (mL) \\
\hline $10+10$ & & 29,5 & 5,0 & 18,0 \\
\hline $20+20$ & 0,3 & 21,3 & 4,0 & 22,5 \\
\hline $30+30$ & & 10,0 & 5,0 & 44,5 \\
\hline $10+10$ & & 32,5 & 3,0 & 16,0 \\
\hline $20+20$ & 0,4 & 29,5 & 4,5 & 22,7 \\
\hline $30+30$ & & 9,8 & 5,0 & 57,0 \\
\hline $10+10$ & & 35,8 & 3,5 & 15,5 \\
\hline $20+20$ & 0,5 & 32,0 & 5,0 & 20,5 \\
\hline $30+30$ & & 8,8 & 3,0 & 71,0 \\
\hline \multicolumn{2}{|c|}{ Especificações (PETROBRAS, 1998b) } & $\geq 15,0$ & $\geq 4,0$ & $\leq 18,0$ \\
\hline
\end{tabular}


Tabela 4. Propriedades dos fluidos preparados com a argila A, aditivados com PHP, degradados e após os tratamentos de proteção e recuperação

Table 4. Properties of drillings fluids prepared with clay called A, treated with PHP, and submitted to degrading additives and after treating of protection and recovery

\begin{tabular}{|c|c|c|c|c|}
\hline $\begin{array}{c}\text { PHP } \\
\text { (g/24,3g de argila) }\end{array}$ & $\begin{array}{c}\mathrm{CaCl}_{2}+\mathrm{MgCl}_{2} \\
\text { (meq/100g de argila) }\end{array}$ & $\mathrm{VA}(\mathrm{cP})$ & $\mathbf{V P}(\mathbf{c P})$ & VF (mL) \\
\hline- & - & 12,5 & 4,8 & 17,7 \\
\hline 0,3 & - & 44,5 & 3,0 & 12,6 \\
\hline 0,4 & - & 50,5 & 5,5 & 11,4 \\
\hline 0,5 & - & 59,8 & 6,0 & 11,4 \\
\hline- & $10+10$ & 10,8 & 4,5 & 17,8 \\
\hline- & $20+20$ & 8,5 & 4,0 & 21,0 \\
\hline- & $30+30$ & 9,7 & 3,8 & 20,5 \\
\hline \multicolumn{5}{|c|}{ Tratamento de Proteção } \\
\hline \multirow{3}{*}{0,3} & $10+10$ & 52,0 & 10,0 & 15,0 \\
\hline & $20+20$ & 51,8 & 12,0 & 14,0 \\
\hline & $30+30$ & 39,3 & 12,0 & 16,2 \\
\hline \multirow{3}{*}{0,4} & $10+10$ & 70,8 & 12,0 & 13,0 \\
\hline & $20+20$ & 63,3 & 15,5 & 13,6 \\
\hline & $30+30$ & 51,5 & 18,0 & 16,4 \\
\hline \multirow{3}{*}{0,5} & $10+10$ & 80,5 & 14,5 & 12,6 \\
\hline & $20+20$ & 74,5 & 12,0 & 11,6 \\
\hline & $30+30$ & 65,5 & 18,5 & 12,0 \\
\hline \multicolumn{5}{|c|}{ Tratamento de Recuperação } \\
\hline $\begin{array}{c}\mathrm{CaCl}_{2}+\mathrm{MgCl}_{2} \\
\text { (meq/100g de argila) }\end{array}$ & $\begin{array}{c}\text { PHP } \\
(\mathrm{g} / 24,3 \mathrm{~g} \text { de argila }) \\
\end{array}$ & $\mathrm{VA}(\mathrm{cP})$ & $\mathrm{VP}(\mathrm{cP})$ & VF (mL) \\
\hline $10+10$ & & 51,5 & 8,0 & 12,4 \\
\hline $20+20$ & 0,3 & 47,0 & 9,0 & 15,4 \\
\hline $30+30$ & & 31,8 & 7,0 & 20,2 \\
\hline $10+10$ & & 58,5 & 6,5 & 14,6 \\
\hline $20+20$ & 0,4 & 55,0 & 10,0 & 13,0 \\
\hline $30+30$ & & 47,3 & 7,0 & 14,0 \\
\hline $10+10$ & & 66,5 & 14,0 & 10,6 \\
\hline $20+20$ & 0,5 & 60,8 & 7,5 & 11,2 \\
\hline $30+30$ & & 67,8 & 14,5 & 11,6 \\
\hline \multicolumn{2}{|c|}{ Especificações (PETROBRAS, 1998b) } & $\geq 15,0$ & $\geq 4,0$ & $\leq 18,0$ \\
\hline
\end{tabular}

Para os fluidos aditivados com CMC BV (Tabela 3) e MIX (Tabela 7), os valores de VA, embora elevados, são muito inferiores aos obtidos quando do tratamento com PHP (Tabela 5). Este comportamento deve-se, muito provavelmente, ao pequeno tamanho das cadeias poliméricas destes aditivos. Embora o MIX seja composto pela mistura do CMC BV e PHP, possuindo assim cadeias poliméricas curtas e longas, quando adicionado aos fluidos confere comportamento reológico próximo ao dos fluidos aditivados com CMC BV.

Segundo Amorim (2003), polímeros de cadeia curta, a exemplo do CMC BV, possuem como principal função reduzir perdas por filtração, apresentando uma ação viscosificante menos pronunciada. Estes polímeros agem geralmente como defloculantes, pois proporcionam uma máxima adsorção das partículas de argila, uma a uma, recobrindo-as e aumentando a distância entre elas (PEREIRA, 2002).

Comparando a ação do CMC BV, do MIX e da PHP, observou-se que os fluidos aditivados com os dois primeiros polímeros apresentaram valores de VA e VP inferiores aos apresentados pelos fluidos aditivados com a PHP, confirmando a teoria acima.

Segundo Pereira (2001), a PHP é um dos polímeros mais utilizados no setor de poços de água por contribuir fortemente na doação de viscosidade ao fluido. Contudo, a incorporação da PHP aos fluidos preparados com as argilas A 
(Tabela 4) e B (Tabela 7), conduziu às dispersões respectivamente aos estados floculado e floculado-gel, provavelmente, devido a formação de pontes. É importante ressaltar que a concentração de argila no fluido é fator determinante, principalmente, quando da aditivação com polímeros de alto peso molecular, como a PHP. Desta forma, concentrações de argila inferiores a utilizada neste estudo podem ser suficientes e em conjunto com a PHP desenvolverem fluidos com viscosidades adequadas. Além disso, a concentração do polímero também afeta diretamente as viscosidades do fluido e se introduzido em excesso pode levar o sistema a um estado de elevado grau de floculação.

Em estudo sobre o efeito de diferentes concentrações de PHP nas propriedades reológicas de sistemas bentonita-água, Gungor e Karaoglan (2001) apresentaram três possibilidades para as interações entre as moléculas de PHP e as partículas de argila: (i) a troca aniônica entre as hidroxilas $\left(\mathrm{OH}^{-}\right)$presentes nas superfícies das partículas de argila e os ânions carboxílicos $\left(\mathrm{COO}^{-}\right)$do polímero, (ii) a formação de ligações hidrogênio entre as hidroxilas da superfície da partícula e o grupo $\mathrm{C}=\mathrm{O}$ do polímero e (iii) o estabelecimento de pontes envolvendo íons divalentes a partir de forças eletrostáticas.

A contaminação dos fluidos preparados com as argilas A (Tabelas 2, 4 e 6) e B (Tabelas 3, 5 e 7) com $\mathrm{CaCl}_{2}+\mathrm{MgCl}_{2}$ provocou modificações nos valores de VA, VP e VF, tornando o sistema com propriedades indesejáveis (segundo a N-2604 da PETROBRAS) para uso em fluidos de perfuração à base de água. Como discutido em trabalho anterior (AMORIM et al., 2004), esta perda de propriedades deve-se à sensibilidade do sistema argila bentonita-água aos íons $\mathrm{Ca}^{2+} \mathrm{e}$ $\mathrm{Mg}^{2+}$ que, quando presentes, inibem a hidratação das partículas de argila, aumentando a força atrativa entre elas, favorecendo interações do tipo face-a-face, ou seja, promovendo a floculação do sistema e, conseqüentemente, a perda das propriedades reológicas. Este comportamento foi mais acentuado para as maiores concentrações do aditivo de contaminação, 20 meq de $\mathrm{CaCl}_{2}+20$ meq de $\mathrm{MgCl}_{2}$ e 30 meq de $\mathrm{CaCl}_{2}+30$ meq de $\mathrm{MgCl}_{2}$. Além disso, a perda das propriedades provocada pela contaminação com $\mathrm{CaCl}_{2}+$ $\mathrm{MgCl}_{2}$ indica que os fluidos poderão ter suas funções comprometidas durante o processo de perfuração. A diminuição de VA e VP indica que o fluido não transportará de forma eficiente até a superfície os detritos gerados pela perfuração, causando uma má limpeza do poço, podendo ocasionar trancamentos e aprisionamentos da coluna de perfuração. Os elevados valores de VF, por sua vez, indicam invasões excessivas de filtrado nas formações geológicas. Como conseqüências, podem ocorrer diversos problemas: desmoronamento das formações hidratáveis, avaliações equivocadas da formação que está sendo perfurada e redução do diâmetro do poço em virtude da formação de rebocos muito espessos, além de contribuir fortemente para o processo de prisão diferencial (AMORIM, 2003).

Para os fluidos preparados com a argila A, o tratamento de proteção mostrou que os aditivos CMC BV (Tabela 2) e MIX (Tabela 6) preservam as propriedades reológicas das dispersões da ação floculante do $\mathrm{Ca}^{2+}$ e do $\mathrm{Mg}^{2+}$, com valores que satisfazem as especificações brasileiras (PETROBRAS, 1998b) para uso em fluidos de perfuração à base de água. Os melhores resultados foram obtidos quando do tratamento com 0,4 e 0,5 g de CMC BV (Tabela 2) e 0,3, 0,4 e $0,5 \mathrm{~g}$ de MIX (Tabela 6) nas três concentrações de $\mathrm{CaCl}_{2}+\mathrm{MgCl}_{2}$. Estes resultados mostraram a eficiência desses aditivos poliméricos como colóide protetor em dispersões de argilas bentoníticas, bem como sua resistência à presença

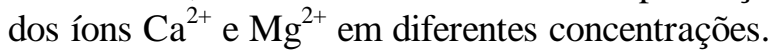
Em contrapartida, o tratamento com a PHP (Tabela 4) conduziu o sistema a um estado de floculação, elevando os valores de VA e diminuindo os valores de VF, não desempenhando comportamento eficiente frente à ação floculante

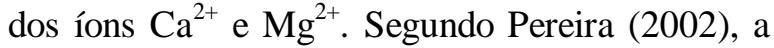
presença de cátions divalentes como $\mathrm{Ca}^{2+} \mathrm{e} \mathrm{Mg}^{2+}$ inibe o alongamento do polímero, que ao invés de desenrolar e expandir permanece unido em forma de novelo, formando aglomerados e diminuindo a sua solubilidade. Através dos resultados obtidos fica evidente que nem todos os polímeros são negativamente influenciados pela presença dos

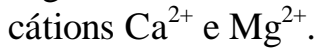

Para os fluidos preparados com a argila B, o tratamento de proteção com os aditivos CMC BV (Tabela 3), PHP (Tabela 5) e MIX (Tabela 7) conduziu o sistema ao estado floculado-gel, caracterizado por elevados valores de VA e baixos valores de VP. O VF também apresentou valores baixos, bem inferiores ao máximo permitido de $18 \mathrm{~mL}$, especificado pela 
PETROBRAS (1998b). Este comportamento pode ser justificado pelo preenchimento dos poros do reboco formado no papel de filtro, durante os ensaios para determinação do VF, pelas cadeias poliméricas não adsorvidas às partículas de argila, como explicam Durand et al. (1997). Desta forma, os aditivos poliméricos estudados não agem como colóide protetor nos fluidos preparados com a argila B.

$\mathrm{O}$ tratamento de reabilitação dos fluidos preparados com a argila $\mathrm{A}$ contaminados com os diferentes teores de $\mathrm{CaCl}_{2}+\mathrm{MgCl}_{2}$ mostrou que o CMC BV (Tabela 2) e o MIX (Tabela 6) recuperam as propriedades reológicas dos fluidos, sendo os melhores resultados obtidos para as concentrações de 0,3, 0,4 e 0,5 g de CMC BV (Tabela 2) e 0,4 e 0,5 g de MIX (Tabela 6), com valores de VA, VP e VF de acordo com os limites especificados pela PETROBRAS (1998b). Neste tratamento, a recuperação das propriedades reológicas e de filtração dos fluidos é promovida pela adsorção do polímero nas partículas de argila, mesmo após a adição do agente de contaminação $\left(\mathrm{CaCl}_{2}+\mathrm{MgCl}_{2}\right)$, aumentando as forças de repulsão e reduzindo/evitando o fenômeno da floculação, provocado pela presença do $\mathrm{Ca}^{2+}$ e do $\mathrm{Mg}^{2+}$ (AMORIM, 2003).

Para os fluidos preparados com a argila B (Tabelas 3, 5 e 7), os resultados obtidos evidenciaram a ação limitada dos três polímeros estudados na recuperação das suas propriedades reológicas e de filtração, principalmente para a concentração de 30 meq de $\mathrm{CaCl}_{2}+30 \mathrm{meq}$ de $\mathrm{MgCl}_{2}$. Para esta concentração de contaminante, observou-se uma redução brusca nos valores de VA e VP e elevação nos valores de VF, indicando a maior sensibilidade do sistema a elevadas concentrações dos cátions floculantes.

Comparando os resultados obtidos para as propriedades reológicas e de filtração dos fluidos estudados, observou-se que, dentre os aditivos poliméricos, o MIX apresentou melhor comportamento frente aos cátions floculantes $\mathrm{Ca}^{2+}$ e $\mathrm{Mg}^{2+}$ em diferentes concentrações, podendo ser utilizado tanto na proteção quanto na recuperação de fluidos hidroargilosos preparados com a argila A. Este resultado indica que não apenas o tipo de polímero, mas também o tipo de argila influencia diretamente na preservação e reabilitação das propriedades reológicas e de filtração dos fluidos à base de água. Além disso, os resultados indicam que no MIX a fração de CMC BV é responsável pela redução das perdas por filtração, enquanto que a fração de PHP responde pela viscosificação do sistema argila bentonítica-água, confirmando as funções dos polímeros de cadeia curta e de cadeia longa. Essa ação conjunta possibilita a obtenção de um aditivo com características adequadas para aplicação em fluidos hidroargilosos, bem como a viabilidade do seu uso como colóide protetor.

A partir deste estudo, foram observadas grandes diferenças no comportamento reológico dos fluidos aditivados com polímeros celulósicos (CMC BV e MIX) e não-celulósicos (PHP). De um modo geral, a aditivação com PHP confere aos fluidos elevadas viscosidades aparente e plástica, sendo atingidos elevados graus de floculação. A aditivação com o CMC BV e com o MIX eleva as viscosidades dos fluidos, contudo, são obtidos fluidos homogêneos com propriedades reológicas e de filtração adequadas, podendo ser utilizados na perfuração de poços de água, de petróleo e em operações de sondagem de minérios. Além disto, observou-se a ação limitada da PHP na proteção e reabilitação dos fluidos contaminados com diferentes concentrações de $\mathrm{CaCl}_{2}+\mathrm{MgCl}_{2}$, ao contrário do $\mathrm{CMC} \mathrm{BV}$ e do MIX que se mostraram eficientes.

\section{CONCLUSÕES}

Com o objetivo de avaliar o efeito de aditivos poliméricos celulósicos e não-celulósicos visando a proteção e reabilitação de fluidos hidroargilosos com diferentes graus de contaminação, concluiuse que:

(i) a aditivação com CMC BV e com MIX corrige o comportamento reológico dos fluidos preparados com a argila A, tornando-os adequados para aplicação na perfuração de poços de água e de petróleo;

(ii) os aditivos CMC BV e MIX agem de forma eficiente na proteção e reabilitação dos fluidos preparados com a argila $\mathrm{A}$ contaminados com diferentes concentrações de $\mathrm{CaCl}_{2}+\mathrm{MgCl}_{2}$;

(iii) a aditivação polimérica e os tratamentos de proteção e reabilitação com a PHP conduzem os fluidos a um estado de elevada floculação, o que é indesejável;

(iv) dentre os aditivos poliméricos estudados, o MIX foi o que apresentou melhor comportamento frente ao agente de contaminação, podendo portanto ser utilizado também como colóide protetor em fluidos hidroargilosos e no MIX, a fração de CMC BV é, provavelmente, responsável 
pela redução das perdas por filtração, enquanto que a fração de PHP responde pela viscosificação do sistema argila bentonítica-água.

Em resumo, a aditivação com PHP confere aos fluidos elevados graus de floculação, enquanto que a aditivação com o CMC BV e com o MIX confere propriedades reológicas e de filtração adequadas. Além disto, a PHP é pouco eficiente na proteção e reabilitação dos fluidos contaminados com diferentes concentrações de cloretos de cálcio e magnésio, ao contrário do CMC BV e do MIX que se mostraram eficientes.

Tabela 5. Propriedades dos fluidos preparados com a argila B, aditivados com PHP, degradados e após os tratamentos de proteção e recuperação

Table 5. Properties of drillings fluids prepared with clay called B, treated with PHP, and submitted to degrading additives and after treating of protection and recovery

\begin{tabular}{|c|c|c|c|c|}
\hline $\begin{array}{l}\text { PHP } \\
\text { (g/24,3g de argila) }\end{array}$ & 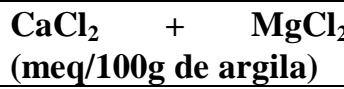 & VA (cP) & $\mathbf{V P}(\mathbf{c P})$ & VF (mL) \\
\hline- & - & 16,1 & 4,0 & 16,3 \\
\hline 0,3 & - & 119,0 & 15,0 & 11,4 \\
\hline 0,4 & - & 133,0 & 19,0 & 10,8 \\
\hline 0,5 & - & 137,8 & 5,5 & 11,4 \\
\hline- & $10+10$ & 16,8 & 3,5 & 19,8 \\
\hline- & $20+20$ & 11,8 & 3,0 & 27,5 \\
\hline- & $30+30$ & 5,0 & 2,5 & 39,0 \\
\hline \multicolumn{5}{|c|}{ Tratamento de Proteção } \\
\hline \multirow{3}{*}{0,3} & $10+10$ & 97,0 & 5,0 & 12,6 \\
\hline & $20+20$ & 52,8 & 16,5 & 14,2 \\
\hline & $30+30$ & 30,3 & 6,5 & 19,6 \\
\hline \multirow{3}{*}{0,4} & $10+10$ & 120,0 & 56,0 & 10,4 \\
\hline & $20+20$ & 51,8 & 14,5 & 13,2 \\
\hline & $30+30$ & 41,5 & 11,0 & 14,8 \\
\hline \multirow{3}{*}{0,5} & $10+10$ & 142,8 & 43,5 & 10,8 \\
\hline & $20+20$ & 101,5 & 53,0 & 10,8 \\
\hline & $30+30$ & 38,5 & 11,5 & 12,0 \\
\hline \multicolumn{5}{|c|}{ Tratamento de Recuperação } \\
\hline $\begin{array}{c}\mathrm{CaCl}_{2}+\mathrm{MgCl}_{2} \\
\text { (meq/100g de argila) }\end{array}$ & $\begin{array}{c}\text { PHP } \\
\text { (g/24,3g de argila) }\end{array}$ & $\mathrm{VA}(\mathbf{c P})$ & $\mathbf{V P}(\mathbf{c P})$ & VF (mL) \\
\hline $10+10$ & \multirow{3}{*}{0,3} & 85,5 & 20,0 & 13,6 \\
\hline $20+20$ & & 30,0 & 6,5 & 13,8 \\
\hline $30+30$ & & 9,3 & 6,0 & 16,2 \\
\hline $10+10$ & \multirow{3}{*}{0,4} & 110,0 & 29,0 & 12,0 \\
\hline $20+20$ & & 30,0 & 8,5 & 13,0 \\
\hline $30+30$ & & 9,8 & 6,5 & 13,0 \\
\hline $10+10$ & \multirow{3}{*}{0,5} & 105,0 & 15,0 & 11,4 \\
\hline $20+20$ & & 31,8 & 14,0 & 11,6 \\
\hline $30+30$ & & 12,3 & 9,0 & 13,0 \\
\hline \multicolumn{2}{|c|}{ Especificações (PETROBRAS, 1998b) } & $\geq 15,0$ & $\geq 4,0$ & $\leq 18,0$ \\
\hline
\end{tabular}


Tabela 6. Propriedades dos fluidos preparados com a argila A, aditivados com MIX, degradados e após os tratamentos de proteção e recuperação

Table 6 Properties of drillings fluids prepared with clay called A, treated with MIX, and submitted to degrading additives and after treating of protection and recovery

\begin{tabular}{|c|c|c|c|c|}
\hline $\begin{array}{c}\text { MIX } \\
(\mathrm{g} / \mathbf{2 4 , 3 g} \text { de argila) } \\
\end{array}$ & $\begin{array}{c}\mathrm{CaCl}_{2}+\mathrm{MgCl}_{2} \\
\text { (meq/100g de argila) }\end{array}$ & VA (cP) & $\mathbf{V P}(\mathbf{c P})$ & VF (mL) \\
\hline- & - & 12,5 & 4,8 & 17,7 \\
\hline 0,3 & - & 15,8 & 6,5 & 17,4 \\
\hline 0,4 & - & 14,5 & 6,5 & 15,0 \\
\hline 0,5 & - & 15,5 & 6,5 & 13,0 \\
\hline- & $10+10$ & 10,8 & 4,5 & 17,8 \\
\hline- & $20+20$ & 8,5 & 4,0 & 21,0 \\
\hline- & $30+30$ & 9,7 & 3,8 & 20,5 \\
\hline \multicolumn{5}{|c|}{ Tratamento de Proteção } \\
\hline \multirow{3}{*}{0,3} & $10+10$ & 19,3 & 6,0 & 21,0 \\
\hline & $20+20$ & 16,5 & 6,0 & 16,6 \\
\hline & $30+30$ & 20,5 & 4,5 & 18,7 \\
\hline \multirow{3}{*}{0,4} & $10+10$ & 19,3 & 6,5 & 15,3 \\
\hline & $20+20$ & 19,8 & 6,5 & 14,5 \\
\hline & $30+30$ & 20,5 & 4,0 & 14,7 \\
\hline \multirow{3}{*}{0,5} & $10+10$ & 21,3 & 6,5 & 13,0 \\
\hline & $20+20$ & 24,0 & 6,0 & 16,2 \\
\hline & $30+30$ & 28,5 & 3,0 & 15,7 \\
\hline \multicolumn{5}{|c|}{ Tratamento de Recuperação } \\
\hline $\begin{array}{c}\mathrm{CaCl}_{2}+\mathrm{MgCl}_{2} \\
\text { (meq/100g de argila) }\end{array}$ & $\begin{array}{c}\text { MIX } \\
\text { (g/24,3g de argila) }\end{array}$ & VA (cP) & $\mathbf{V P}(\mathbf{c P})$ & VF (mL) \\
\hline $10+10$ & & 13,8 & 6,0 & 21,0 \\
\hline $20+20$ & 0,3 & 13,8 & 5,0 & 18,4 \\
\hline $30+30$ & & 18,0 & 5,0 & 18,7 \\
\hline $10+10$ & & 19,3 & 6,5 & 15,5 \\
\hline $20+20$ & 0,4 & 18,5 & 5,5 & 15,0 \\
\hline $30+30$ & & 20,3 & 5,0 & 15,5 \\
\hline $10+10$ & & 22,5 & 6,0 & 15,5 \\
\hline $20+20$ & 0,5 & 27,5 & 4,5 & 16,2 \\
\hline $30+30$ & & 19,3 & 6,0 & 15,5 \\
\hline \multicolumn{2}{|c|}{ Especificações (PETROBRAS, 1998b) } & $\geq 15,0$ & $\geq 4,0$ & $\leq 18,0$ \\
\hline
\end{tabular}


Tabela 7. Propriedades dos fluidos preparados com a argila B, aditivados com MIX, degradados e após os tratamentos de proteção e recuperação

Table 7. Properties of drillings fluids prepared with clay called B, treated with MIX, and submitted to degrading additives and after treating of protection and recovery

\begin{tabular}{|c|c|c|c|c|}
\hline $\begin{array}{c}\text { MIX } \\
\text { (g/24,3g de argila) }\end{array}$ & $\begin{array}{c}\mathrm{CaCl}_{2}+\mathrm{MgCl}_{2} \\
\text { (meq/100g de argila) }\end{array}$ & VA (cP) & $\mathbf{V P}(\mathbf{c P})$ & VF (mL) \\
\hline- & - & 16,1 & 4,0 & 16,3 \\
\hline 0,3 & - & 27,5 & 6,0 & 14,5 \\
\hline 0,4 & - & 34,0 & 5,5 & 20,0 \\
\hline 0,5 & - & 41,3 & 4,5 & 14,0 \\
\hline- & $10+10$ & 16,8 & 3,5 & 19,8 \\
\hline- & $20+20$ & 11,8 & 3,0 & 27,5 \\
\hline- & $30+30$ & 5,0 & 2,5 & 39,0 \\
\hline \multicolumn{5}{|c|}{ Tratamento de Proteção } \\
\hline \multirow{3}{*}{0,3} & $10+10$ & 44,0 & 2,5 & 16,5 \\
\hline & $20+20$ & 44,0 & 2,0 & 17,0 \\
\hline & $30+30$ & 30,3 & 3,5 & 20,0 \\
\hline \multirow{3}{*}{0,4} & $10+10$ & 46,8 & 3,0 & 15,5 \\
\hline & $20+20$ & 51,5 & 4,0 & 16,5 \\
\hline & $30+30$ & 30,5 & 3,0 & 19,7 \\
\hline \multirow{3}{*}{0,5} & $10+10$ & 53,0 & 1,5 & 15,5 \\
\hline & $20+20$ & 51,0 & 6,5 & 16,0 \\
\hline & $30+30$ & 28,0 & 3,5 & 19,0 \\
\hline \multicolumn{5}{|c|}{ Tratamento de Recuperação } \\
\hline $\begin{array}{c}\mathrm{CaCl}_{2}+\mathrm{MgCl}_{2} \\
\text { (meq/100g de argila) }\end{array}$ & $\begin{array}{c}\text { MIX } \\
\text { (g/24,3g de argila) }\end{array}$ & VA (cP) & $\mathbf{V P}(\mathbf{c P})$ & VF (mL) \\
\hline $10+10$ & & 33,9 & 2,7 & 16,9 \\
\hline $20+20$ & 0,3 & 22,0 & 4,3 & 21,0 \\
\hline $30+30$ & & 6,8 & 3,5 & 29,0 \\
\hline $10+10$ & & 41,0 & 2,0 & 16,7 \\
\hline $20+20$ & 0,4 & 22,5 & 5,5 & 21,0 \\
\hline $30+30$ & & 7,3 & 3,0 & 29,0 \\
\hline $10+10$ & & 46,8 & 4,0 & 16,0 \\
\hline $20+20$ & 0,5 & 23,3 & 4,5 & 19,5 \\
\hline $30+30$ & & 8,3 & 4,0 & 30,0 \\
\hline \multicolumn{2}{|c|}{ Especificações (PETROBRAS, 1998b) } & $\geq 15,0$ & $\geq 4,0$ & $\leq 18,0$ \\
\hline
\end{tabular}




\section{REFERÊNCIAS}

ACCIOLY, C.V. Uso de Argilas Esmectíticas em Lamas para Execução de Paredes de Diafragma. Campina Grande, PB, 1994. Dissertação (Mestrado em Engenharia Civil). Departamento de Engenharia Civil - UFPB.

AMORIM, L.V. Melhoria, Proteção e Recuperação da Reologia de Fluidos Hidroargilosos para Uso na Perfuração de Poços de Petróleo. Campina Grande, 2003. Tese (Doutorado em Engenharia de Processos). Universidade Federal de Campina Grande - UFCG.

AMORIM, L.V.; GOMES, C.M.; VIANA, J.D.; FARIAS, K.V.; FERREIRA, H.C. Uso do CMC como Colóide Protetor em Fluidos de Perfuração à Base de Água e Argila. Anais XV CBECIMAT, Natal, p. 1246-1252, 2002.

AMORIM, L.V.; VIANA, J.D.; FARIAS, K.V.; FRANÇA, K.B.; LIRA, H.L.; FERREIRA, H.C. Uso do CMC como Colóide Protetor em Fluidos de Perfuração à Base de Água e Argilas: Parte II. In: Anais $2^{\circ}$ Congresso Brasileiro de P \& D em Petróleo e Gás, Rio de Janeiro, 2003.

AMORIM, L.V.; PEREIRA, E.; GOMES, C.M.; VIANA, J.D.; FARIAS, K.V.; BARBOSA, M.I. R.; FRANÇA, K.B.; LIRA, H.L.; FERREIRA, H.C. Aditivos Poliméricos como Fator de Proteção e Reabilitação em Fluidos Hidroargilosos. Águas Subterrâneas, São Paulo, n. 18, p. 9-18, 2004.

BARBOSA, M.I.R.; VIANA, J.D.; FARIAS, K.V.; PEREIRA, E.; AMORIM, L.V.; LIRA, H.L.; FERREIRA, H.C. Polymeric additives effects in rheological properties of bentonite clay dispersions from Paraíba, Brazil. In: 8th INTERNATIONAL CONGRESS ON APPLIED MINERALOGY (2004, Águas de Lindóia). Proceedings..., v. 2, p. 657-660, 2004a.

BARBOSA, M.I.R.; VIANA, J.D.; FARIAS, K.V.; PEREIRA, E.; AMORIM, L.V., FERREIRA, H. C. Estudo de Aditivos Poliméricos À Base de Celulose na Proteção e Reabilitação de Fluidos Hidroargilosos. Anais XV Congresso Brasileiro de Engenharia Química, Curitiba, 2004b.

DURAND, C., LECOURTIER, J., ROSENBERG, E.; LOEBER, L. Relationship Between Composition, Structure and Permeability of Drilling Filter Cakes, Revue de I'Institute Français du Pétrole n. 51, v. 06, p. 777-788, 1997.

FARIAS, K.V. Estudo de Aditivos Protetores Visando uma Maior Durabilidade de Fluidos de Perfuração à Base de Água e Argila. Relatório Final de Iniciação Científica PIBIC/CNPq, Campina Grande: 2003. DEMA/CCT/UFCG, Projeto Concluído.
GUNGOR, N.; KARAOGLAN, S. Interaction of Polyacrylamide Polymer with Bentonite in Aqueous Systems, Materials Letters n. 48, p. 168-175, 2001.

LUCKHAM, P.F.; ROSSI, S., The Colloidal and Rheological Properties of Bentonite Suspension, Advances in Colloid and Interface Science, n. 82, p. 43-92, 1999.

OLIVEIRA, I.R.; STUDART, A.R.; PILEGGI, R.G.; PANDOLFELLI, V.C. Dispersão e Empacotamento de partículas, princípios e aplicações em processamento cerâmico, São Paulo: Fazendo Arte Editorial, 2000.

PEDROSO, M.A.S. Modificações de Propriedades Reológicas de Dispersões de Argilas Esmectíticas Sódicas. Campina Grande, PB, 1991. Dissertação (Mestrado em Engenharia Química). Departamento de Engenharia Química - UFPB.

PEREIRA, E. O Uso de Inibidores de Argilas como Soluções em Problemas de Sondagem, Conferência proferida durante o XII Encontro de Perfuradores de Poços/XI Simpósio de Hidrogeologia do Nordeste, Anais, Olinda, PE, p. 03-20, 2001.

PEREIRA, E. Química dos Polímeros e Aplicações - Parte I, II, III e IV, 2002. (Disponível em: Www.systemmud.com.br)

PETROBRAS, Argila ativada para fluido de perfuração à base de água na exploração e produção de petróleo, Método, N-2605, 1998a.

PETROBRAS, Argila ativada para fluido de perfuração à base de água na exploração e produção de petróleo, Especificação, N-2604, 1998b.

SOMASUNDARAN, P.; HEALY, T.W.; FUERSTENAU, D.W. The Aggregation of Colloidal Alumina Dispersion by Adsorbed Surfactant Ions, Journal of Colloid and Interface Science, n. 22, v. 6, p. 599-605, 1996.

\section{AGRADECIMENTOS}

Os autores agradecem ao CNPq pela bolsa DCR, Processo N ${ }^{\mathrm{o}}$ 309873/2003-7, à System Mud Indústria e Comércio Ltda. pelo fornecimento dos aditivos poliméricos e ao Laboratório de Referência em Dessalinização - LABDES pelo uso de suas instalações e apóio à pesquisa. 
O uso de polímeros no tratamento e recuperação de fluidos de perfuração contaminados 